\title{
Fuzzy Control-Based Energy-Aware Routing Protocol for Wireless Body Area Networks
}

\author{
Xintong Wang $\mathbb{D}^{1},{ }^{1}$ Guoqiang Zheng $\mathbb{D}^{1,2}$ Huahong Ma, ${ }^{1}$ Weiwei Bai, ${ }^{1}$ Honghai Wu, ${ }^{1}$ \\ and Baofeng Ji \\ ${ }^{1}$ School of Electronic Information Engineering, Henan University of Science and Technology, Luoyang 471023, China \\ ${ }^{2}$ Henan Key Laboratory for Machinery Design and Transmission System, Henan University of Science and Technology, \\ Luoyang 471003, China
}

Correspondence should be addressed to Guoqiang Zheng; lyzgq@haust.edu.cn

Received 25 September 2020; Revised 14 December 2020; Accepted 18 December 2020; Published 6 January 2021

Academic Editor: Eduard Llobet

Copyright ( 2021 Xintong Wang et al. This is an open access article distributed under the Creative Commons Attribution License, which permits unrestricted use, distribution, and reproduction in any medium, provided the original work is properly cited.

\begin{abstract}
Advances in medical and communication technologies have empowered the development of Wireless Body Area Networks (WBANs). WBANs interconnect with miniature sensors placed on the human body to enable medical monitoring of patient health. However, the limited battery capacity, delay, and reliability of data transmission have brought challenges to the wider application of WBAN. Minimum consumption of energy and maximum satisfaction with the QoS requirements are essential design aims of the WBAN schemes. Therefore, a fuzzy control-based energy-aware routing protocol (EARP) is proposed in this paper, the proposed protocol establishes a fuzzy control model composed of remaining node energy and link quality, and the best forwarder node is determined by the processes of fuzzification, fuzzy inference, and defuzzification. The simulation results showed that compared with the performance of the existing EERDT and M-TSIMPLE protocols, the proposed EARP has better performance, including extending network lifetime and improving the reliability of data transmission.
\end{abstract}

\section{Introduction}

With the continuous development of intelligent health, Wireless Body Area Network (WBAN), which is extended from Wireless Sensor Networks (WSNs) [1, 2], has attracted much attention [3]. WBAN is a cross-technology field, which is related to sensor technology, short-distance wireless communication technology, and WSN. Based on these technologies, WBAN has its unique characteristics. The short-distance communication technology applied to WBAN requires lower power at the same data transmission rate. For sensors implanted into the human body, miniaturized, lowpower, and intelligent sensors are the basis to support WBAN. Although WBAN is a part of WSN, WBAN is different from WSN in that it is characterized by small network scale, difficult battery charging, and high biocompatibility.

WBAN is primarily applied in telemedicine, remote monitoring and other health care fields [4]. Figure 1 depicts a basic WBAN architecture. Multiple sensor nodes and a sink are deployed on the human body. The sensor node is utilized to sense the physiological data and send these sensory data to sink; these data are received by sink and sent to the external network terminal after integrated processing [5]. The function of the network terminal is to use these data to monitor or diagnose the patient's condition.

Data collection and transmission rely on technologies such as hardware specifications, MAC layer, and routing $[6,7]$. WBAN needs to quickly and efficiently forward the data sensed by the sensors to the medical service terminal. Routing technology is to solve the problem of data transmission in the network [8].

Sensor nodes need to be comfortable and convenient whether deployed on or in the body; its small size leads to limited battery capacity. A sensor consumes the most energy by a communication process [9]; therefore, energy efficiency of node can be effectively utilized and is a substantial way to ensuring more data transmission under limited resource conditions $[10,11]$. Sensors and sink may not communicate 


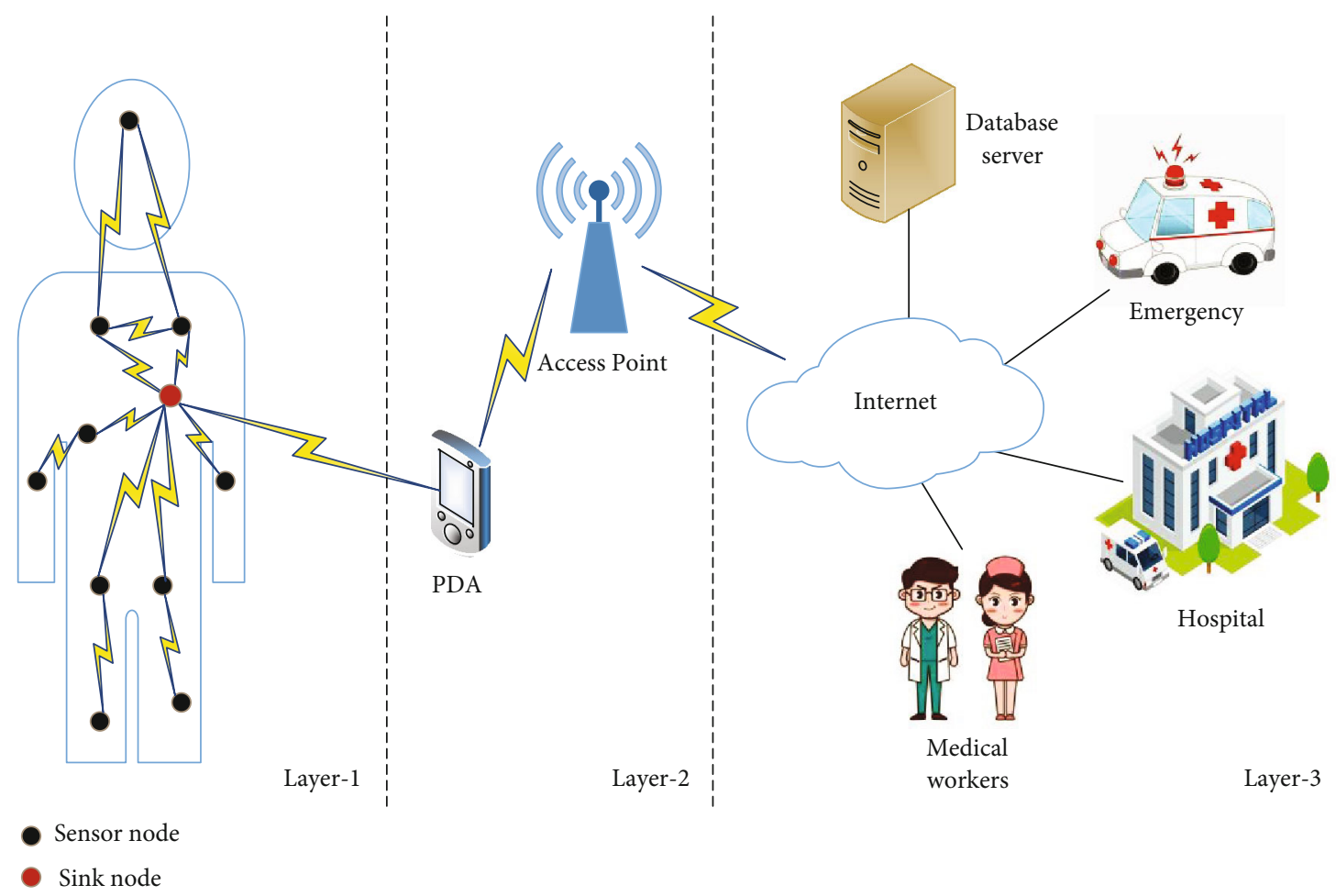

FIGURE 1: Architecture of WBAN.

directly due to the dynamic characteristics of WBANs, so multihop communication is more suitable for WBANs in energy-saving research [12]. The appropriate forwarder node selection is vital in multihop communication [13]. However, existing multihop routing protocols attempt to consider the shortest path as the main influence factor when selecting forwarder node, ignoring the impact of link quality, which results in high power consumption in WBANs. Furthermore, the QoS requirements of different data should be met to comply with the characteristics of WBAN [14, 15], and the calculation of path cost also needs a logical method to achieve.

Hence, we propose a fuzzy control based energy-aware routing protocol, the novelty of this method is that it considers the mobility of sensor nodes and establishes a fuzzy control model with the parameters of residual energy and link quality, and the best forwarder node is determined logically by the processes of fuzzification, fuzzy inference, and defuzzification. This method can ultimately improve the overall performance of the network.

The main contributions of this paper are summarized below.

(i) In a related work, the comparative table is utilized to analyze the current related researches

(ii) Residual energy and link quality are considered to establish a fuzzy control model, and the output value of the model is logically determined as the best forwarder node

(iii) Data were divided into emergency data and periodic data in this paper, and the corresponding forwarder node is selected depend on different data types for data transmission

The rest of this article is organized as follows: Section 2 introduces a related work. Section 3 introduces the system model. Section 4 describes the EARP protocol design in detail. Section 5 depicts the performance of EARP protocol through simulation. Section 6 is the summary of this paper.

\section{Related Work}

The application scenario of WBAN is special, and energy is limited. How to ensure timely and effective data transmission to the network terminal under the limited energy is the focus of routing protocol research. Multihop transmission has the characteristics of balancing network energy consumption; researchers nowadays are more focused on developing a routing mechanism for WBAN next-hop node selection. In this section, we mainly introduce the energy-based multihop routing protocol [16-19].

Javaid et al. [20] proposed M-ATTEMPT targets to improve network energy consumption and reduce the generation of hotspots. This protocol directly transmits sensitive and on-demand data, while normal data adopts multihop transmission. For multihop transmission, the best forwarder node selected has the characteristics of less hops and high residual energy. M-ATTEMPT reduces heat generated and prolongs the network lifetime. However, when a node receives a packet and its temperature exceeds the threshold, the packet is retransmitted, which causes more energy consumption and has low network reliability [21]. 
Since the dynamic characteristics of WBANs, Javaid et al. [22] proposed an improved stable increased-throughput multihop link efficient routing protocol (iM-SIMPLE). This protocol exploits the parameters such as the remaining node energy and the distance of the sink to construct a cost function to select the forwarder node. This protocol performs well in network lifetime. However, in iM-SIMPLE, the nodes that generate critical data are placed near the sink; these nodes are usually selected as forwarder nodes, which results in the failure of sending the critical data and reduced network reliability.

Moid et al. [23] proposed a routing protocol for balanced energy consumption (BEC). The protocol employs residual energy to establish a cost function and selects the neighbor node with the minimum cost function as the forwarder to transmit data. Moreover, the protocol sets an energy threshold, when the node energy is lower than the threshold; the node only forwards critical data, which can effectively balance the energy consumption in the network, but the selected forwarder nodes consume much energy due to the important tasks assigned.

The authors in [24] presented a cluster-based routing protocol, named energy efficient and reliable data transfer (EERDT) protocol for WBAN, which selects cluster heads of each cluster depending on the cost efficiency function represented by the ratio of residual energy and initial energy and uses cluster heads to transmit data. This protocol improves energy efficiency.

In [25], the authors proposed a mobility supported threshold based stability increased throughput to sink using multihop routing protocol (M-TSIMPLE). The protocol divides the node types into child nodes and parent nodes depending on the network flow tree structure. The child node selects the appropriate parent node as the forwarder node to transmit data by the cost function value; the parent node receives and forwards the data to sink. Moreover, if the node's energy is below a threshold, the node will not participate in any forwarding. The protocol utilizes threshold control to save energy consumption. However, the link quality between child node and parent node is poor due to the mobility of human body.

Anwar et al. [26] introduced a green communication framework focusing on an energy aware link efficient routing approach for WBANs (ELR-W). This protocol takes into account the four parameters of residual energy, link efficiency, node to coordinator distance, and hop count to construct a path cost model, which is used to select the next-hop node for transfer data. This method can effectively prolong the network lifetime, but it is not suitable for the transmission of different data types.

Qureshi et al. [27] proposed ELA-RP targets to save energy in WBANs; this protocol calculates the energy level and link quality of neighbor nodes to select the best next hop node. Moreover, the energy consumed by the HELLO message is part of the total energy consumption. The source node sends out a control HELLO message packet to control the propagation of HELLO messages in the network. The scheme balances the energy consumption.

In [28], TCBR is proposed, which is an optimal trust aware cluster based routing protocol. The authors use an improved evolutionary particle optimization algorithm (IEPSO) to select cluster heads in different clusters. The fuzzy inference model composed of residual energy, link failure time, and received signal strength is used to select the appropriate routing path. Compared with the traditional cluster-based routing protocol, this protocol achieves better cluster-based performance in energy saving, network lifetime, and throughput.

Qureshi et al. [29] proposed link quality and energy utilization-based preferable next hop selection routing. The protocol evaluates the remaining energy and link quality of the nodes, which are used as decision parameters for selecting the next hop node. Moreover, the proposed protocol adopts handshake mechanism by using control messages to reduce network overhead. This method achieves load balancing, but it is not suitable for different WBAN scenarios.

The above-mentioned related protocols are summarized in Table 1 . These routing protocols combine mutually independent parameters together to calculate the path cost by model or function and select the forwarder node depending on the path cost value. Moreover, it is found from relevant protocols that transmission by various data types satisfies different QoS requirements, and the reliability is affected by link quality. Hence, we propose a fuzzy control-based energyaware routing protocol, which comprehensively considers multiple parameters such as hop count, remaining node energy, and link quality and establishes a fuzzy control model consisting of the remaining node energy and link quality; the best forwarder node is determined by the processes of fuzzification, fuzzy inference, and defuzzification, eventually the method to enhance the overall performance of the network.

\section{System Model}

3.1. Network Model. In this section, the design of proposed protocol discusses with some assumptions and its model.

The WBAN network model with $N$ sensor nodes and one sink node is exhibited in Figure 2; the sink is located at the waist of the human body. Sensor nodes are deployed on the body surface; they are mainly responsible for data monitoring and transmit the collected data directly or through intermediate nodes to sink. The sink node is composed of mobile intelligent devices; it is the hub between WBAN and external network, mainly taking care of forwarding data.

This paper also defines two types of nodes: a static node and mobile node. The static node indicates that the sensor node is placed at a location that has nothing to do with the movement of the human body, while the mobile node represents that the sensor is placed in the limbs of the human body; for the data delivery on the mobile node, store and carry scheme are employed.

The following are the model assumptions:

(i) All sensor nodes are placed in different positions of the human body to collect corresponding physiological information and have specific ID

(ii) All sensor nodes have the same initial energy and transmission range; the maximum transmission range is $l_{\max }$ 
TABLE 1: Comparison of related protocols.

\begin{tabular}{|c|c|c|c|c|c|}
\hline Protocols & Parameters & Methodology & $\begin{array}{c}\text { Energy } \\
\text { conservation }\end{array}$ & Reliability & Shortcomings \\
\hline M-ATTEMPT & Hop count and residual energy & $\begin{array}{l}\text { Linear programming } \\
\text { model }\end{array}$ & $\sqrt{ }$ & & $\begin{array}{l}\text { Retransmitted power causes } \\
\text { energy consumption }\end{array}$ \\
\hline iM-SIMPLE & Distance and residual energy & Cost function & $\sqrt{ }$ & & $\begin{array}{c}\text { Not suitable for emergency } \\
\text { applications }\end{array}$ \\
\hline $\mathrm{BEC}$ & Residual energy & Cost function & $\sqrt{ }$ & & $\begin{array}{l}\text { Selected forwarder nodes } \\
\text { consume much energy }\end{array}$ \\
\hline EERDT & Residual energy and initial energy & Cost efficient function & $\sqrt{ }$ & & Network adaptability \\
\hline M-TSIMPLE & Distance and residual energy & $\begin{array}{l}\text { Network flow tree } \\
\text { structure and cost function }\end{array}$ & $\sqrt{ }$ & & $\begin{array}{l}\text { Mobility causes unstable } \\
\text { link connection }\end{array}$ \\
\hline ELA-W & $\begin{array}{l}\text { Residual energy, link efficiency, } \\
\text { distance, and hop count }\end{array}$ & Path cost model & $\sqrt{ }$ & $\sqrt{ }$ & $\begin{array}{l}\text { Not suitable for different } \\
\text { data types of transfer }\end{array}$ \\
\hline ELA-RP & Remaining node energy, link quality & Score function & $\sqrt{ }$ & $\sqrt{ }$ & Unconsidered reliability \\
\hline TCBR & $\begin{array}{l}\text { Residual energy, link expiration time, } \\
\text { and received signal strength }\end{array}$ & $\begin{array}{l}\text { Fuzzy-based trust } \\
\text { inference model }\end{array}$ & $\sqrt{ }$ & $\sqrt{ }$ & $\begin{array}{l}\text { Algorithm increases } \\
\text { computing time }\end{array}$ \\
\hline ERP & Residual energy and link quality & Score function & $\sqrt{ }$ & $\sqrt{ }$ & $\begin{array}{l}\text { Not suitable for different } \\
\text { scenarios }\end{array}$ \\
\hline
\end{tabular}

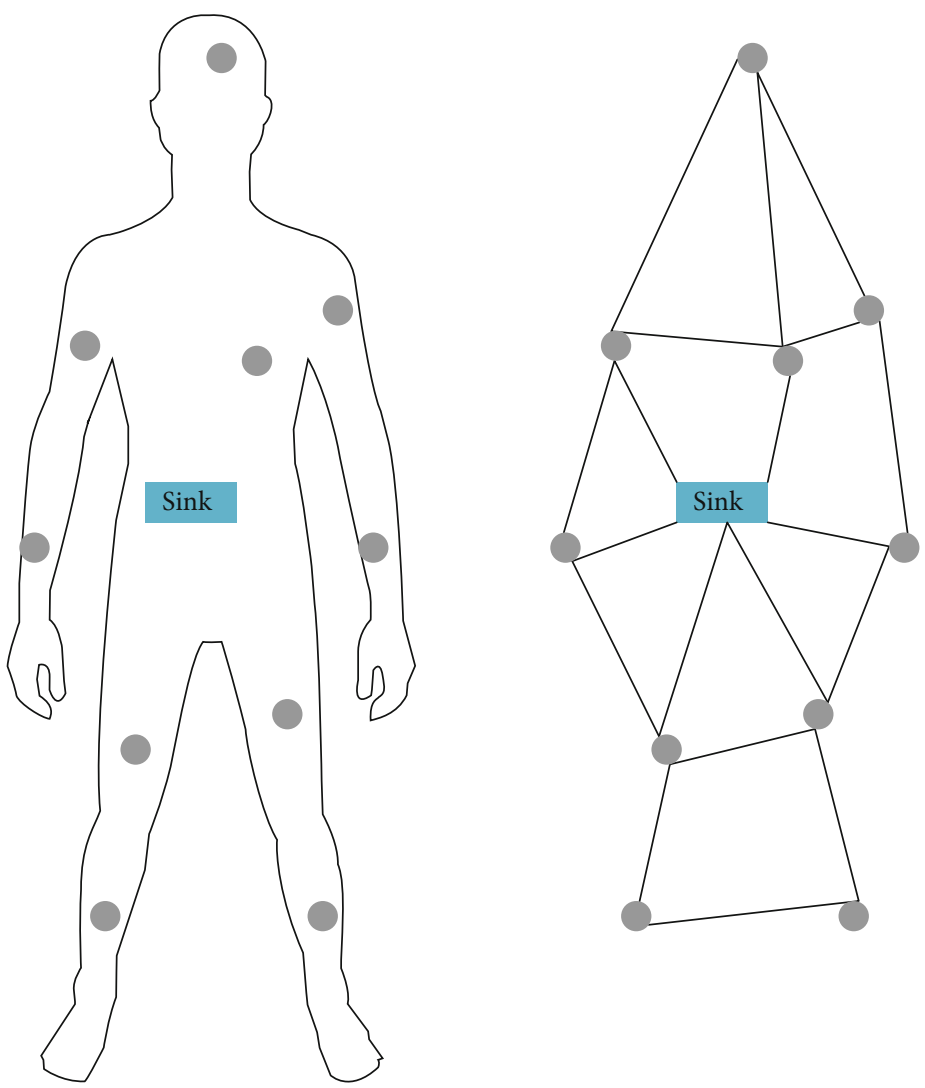

Figure 2: Network topology.

(iii) Sink node has strong information processing capability; it only receives data from the sensor and does not generate packet itself. Moreover, we do not regard the energy of the sink

(iv) Due to the dynamic nature of WBAN, the one-hop star network topology cannot guarantee reliable data transmission. Hence, the proposed protocol adopts a multihop network topology, and the maximum number of hops is three

Since the sensor nodes are deployed in different locations of the human body and the importance of the physiological data sensed is different, the physiological data can be divided 
into periodic data and emergency data and these two types of data are prioritized; emergency data has higher priority than periodic data.

(1) Periodic data: it refers to the periodic transmission packets generated by node. The packets are transmitted to sink periodically, and the traffic is large, but the realtime performance of packets transmission is not high

(2) Emergency data: abnormal packets exceed the normal health value, which is nonperiodic packets generated by nodes and has low data traffic. If the emergency data are not effectively transmitted, it is likely to endanger human life and health. Therefore, the real-time and reliable transmission requirements for emergency data are high

3.2. Radio Model. In WBAN, each sensor node consumes energy in the process of data perception, processing, and transmission, but the energy consumed by the data transmission process accounts for the largest proportion. Therefore, this protocol mainly analyzes the energy consumption of node in data transmission process and uses a first-order radio model to calculate the energy consumption [30], which is calculated as follows:

$$
\begin{aligned}
E_{\mathrm{tx}}(n, d) & =E_{\mathrm{tx}-\mathrm{elec}}(n)+E_{\mathrm{amp}}(n, d), \\
E_{\mathrm{tx}}(n, d) & =E_{\mathrm{tx}-\mathrm{elec}} \times n+E_{\mathrm{amp}} \times n \times d^{2}, \\
E_{\mathrm{rx}}(n) & =E_{\mathrm{rx}-\mathrm{elec}}(n), \\
E_{\mathrm{rx}}(n) & =E_{\mathrm{rx}-\mathrm{elec}} \times n,
\end{aligned}
$$

where $n$ indicates the size of the data packet; $d$ is the distance between the sender and the receiver; $E_{\mathrm{tx}}(n, d)$ is composed of the energy consumption of the internal circuit $E_{\text {tx-elec }}$ and signal amplifier $E_{\text {amp }}$, which represents the energy consumption of transmitting data; and $E_{r x}(n)$ represents the energy consumed by the received data. The receiver does not need to amplify the signal only, i.e., $E_{\text {rx-elec }}$, as the energy consumption of the internal circuit is required.

\section{EARP: Protocol Description}

4.1. Proposed Parameters. The proposed protocol mainly considers parameters such as residual energy, link quality, and hop count.

Since the limited sensor energy in WBAN, we need to consider residual energy when selecting the forwarder node. Moreover, data loss and delay are easy to be caused due to the dynamic characteristics in WBAN; link quality and hop count should also be regarded to ensure the timeliness and reliability of data transmission.

(1) Residual energy: it refers to the total energy minus the consumed energy, and its normalization calculation is as follows:

$$
\mathrm{RE}_{i}=\frac{E_{\text {res }}-E_{\text {th }}}{E_{\text {initial }}-E_{\text {th }}},
$$

where $R E_{i}$ represents the normalized residual energy of node $i, E_{\text {initial }}$ is the initial energy, $E_{\mathrm{th}}$ is the energy threshold, and $E_{\text {res }}$ indicates the residual energy

(2) Link quality: the link quality depends on the received signal strength (RSSI). RSSI can be computed in

$$
\mathrm{RSSI}=10 \log \frac{P_{\mathrm{rx}}}{P_{\mathrm{tx}}},
$$

RSSI is defined as the ratio of the received power to the transmitted power, $P_{\mathrm{rx}}$ denotes the received power, and $P_{\mathrm{tx}}$ is the transmitted power. Normalized link quality $L Q_{i}$ between node $i$ and $j$ which was carried by HELLO packet; this value is computed by equation (4), where RSSI ${ }_{\text {min }}$ represents the minimum signal strength for data packet transmission.

$$
\mathrm{LQ}_{i}=\frac{\mathrm{RSSI}_{i, j}-\mathrm{RSSI}_{\text {min }}}{\mathrm{RSSI}_{i, j}}
$$

(3) Hop count: hop count is the number of hops from the node to sink. In the initialization phase, this value is updated by exchanging HELLO packets between neighbor nodes.

4.2. Path Benefit Calculation. The residual energy (RE) and link quality (LQ) parameters of the candidate forwarder nodes are utilized to calculate the path benefit value through the process of fuzzification, fuzzy inference, and defuzzification. The candidate forwarder node with the maximum path benefit $(\mathrm{PB})$ is selected as the best forwarder node. Figure 3 depicts the fuzzy control process.

Where residual energy and link quality are input variables, the fuzzy inputs re and lq are obtained by fuzzing the input variables, the fuzzy output $\mathrm{pb}$ is obtained by a fuzzy inference process, and PB is obtained by defuzzification.

4.2.1. Fuzzification. Both input variables and output variables belong to the control variables of implementing fuzzy control. Neither input variables nor output variables can be directly used to perform fuzzy control. Therefore, the control variables must be fuzzed.

First, the input variables are fuzzified; the results of the fuzzification are expressed in fuzzy language $\{L, M, H\}$ (low, medium, high). If the more fuzzy languages of the input variables are arranged, the fuzzy rules would increase, which lead to the increase of model calculation time. Therefore, RE and LQ are quantified into $\{L, M, H\}$. Since the residual energy and link quality have been normalized, the domain of residual energy is $R E=[0,1]$, and the domain of link quality is $\mathrm{LQ}=[0,1]$.

Each fuzzy language has its corresponding membership function; triangular membership function is adopted for residual energy and link quality. The membership function represents the membership degree of fuzzy variables to fuzzy sets. The closer the value is to 1 , the higher the membership degree of the fuzzy variable. The membership function of residual energy is established according to the fuzzy domain, 


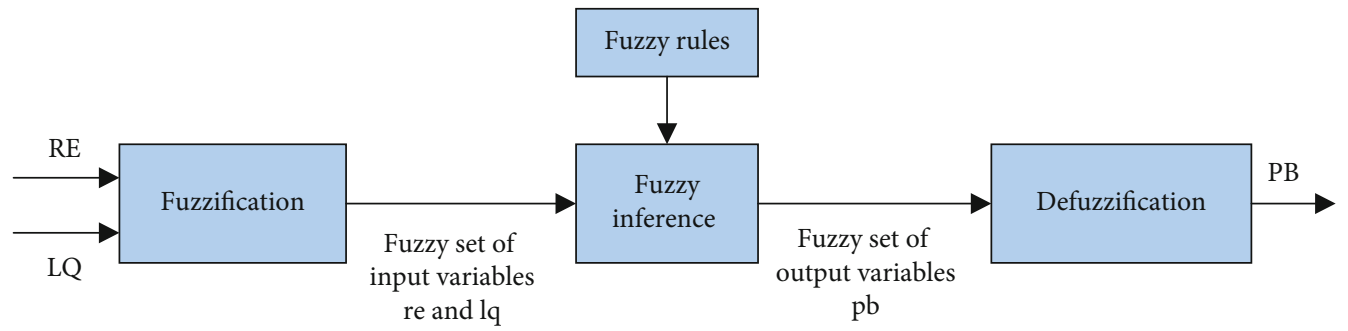

FIgURe 3: Fuzzy control process.

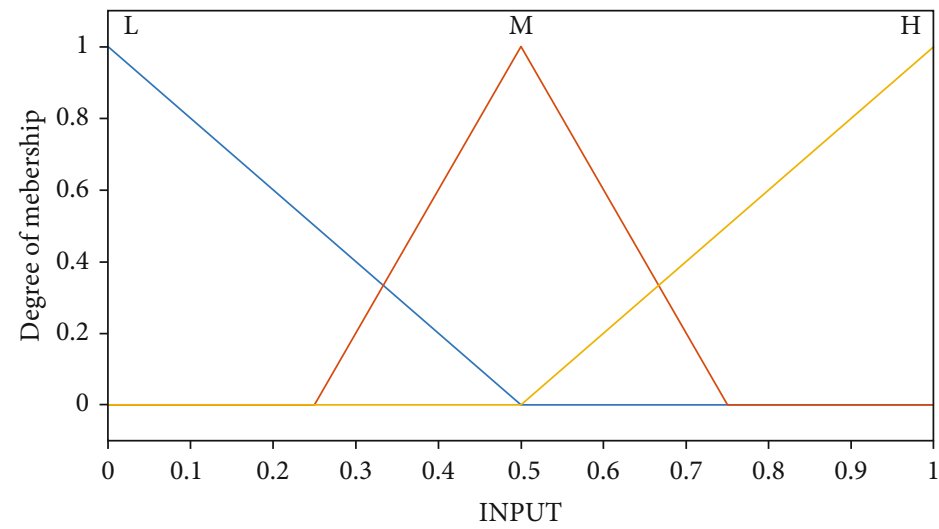

FIgURE 4: Membership function curve of input variables.

as shown in equations (5)-(7), and the membership function of link quality is computed in equations (8)-(10).

$$
\begin{aligned}
& \mu_{\mathrm{L}}(\mathrm{RE})=\left\{\begin{array}{l}
-2 \mathrm{RE}+1,0 \leq \mathrm{RE} \leq 0.5, \\
0, \mathrm{RE}>0.5 \& \mathrm{RE}<0,
\end{array}\right. \\
& \mu_{\mathrm{M}}(\mathrm{RE})=\left\{\begin{array}{l}
-4 \mathrm{RE}-1,0.25 \leq \mathrm{RE} \leq 0.5, \\
-4 \mathrm{RE}+3,0.5 \leq \mathrm{RE} \leq 0.75, \\
0, \mathrm{RE}>0.25 \& \mathrm{RE}>0.75,
\end{array}\right. \\
& \mu_{\mathrm{H}}(\mathrm{RE})=\left\{\begin{array}{l}
4 \mathrm{RE}-3,0.75 \leq \mathrm{RE} \leq 1, \\
0, \mathrm{RE}<0.75 \& \mathrm{RE}>1,
\end{array}\right. \\
& \mu_{\mathrm{L}}(\mathrm{LQ})=\left\{\begin{array}{l}
-2 \mathrm{LQ}+1,0 \leq \mathrm{LQ} \leq 0.5, \\
0, \mathrm{LQ}>0.5 \& \mathrm{LQ}<0,
\end{array}\right. \\
& \mu_{\mathrm{M}}(\mathrm{LQ})=\left\{\begin{array}{l}
-4 \mathrm{LQ}-1,0.25 \leq \mathrm{LQ} \leq 0.5, \\
-4 \mathrm{LQ}+3,0.5 \leq \mathrm{LQ} \leq 0.75, \\
0, \mathrm{LQ}>0.25 \& \mathrm{LQ}>0.75,
\end{array}\right. \\
& \mu_{\mathrm{H}}(\mathrm{LQ})=\left\{\begin{array}{l}
4 \mathrm{LQ}-3,0.75 \leq \mathrm{LQ} \leq 1, \\
0, \mathrm{LQ}<0.75 \& \mathrm{LQ}>1,
\end{array}\right.
\end{aligned}
$$

where $\mu_{\mathrm{L}}(\mathrm{RE})$ represents the membership function of RE belonging to $\mathrm{L}, \mu_{\mathrm{M}}(\mathrm{RE})$ is the membership function of $\mathrm{RE}$ belonging to $\mathrm{M}, \mu_{\mathrm{H}}(\mathrm{RE})$ indicates the membership function of RE belonging to $H, \mu_{L}(L Q)$ is the membership function of LQ belonging to $\mathrm{L}, \mu_{\mathrm{M}}(\mathrm{LQ})$ is the membership function of LQ belonging to $\mathrm{M}$, and $\mu_{\mathrm{H}}(\mathrm{LQ})$ represents the membership function of LQ belonging to $H$. The representation principle of membership function means that the membership functions of variables should be distributed reasonably in the domain; that is, they should cover the whole domain, symmetric and balanced.

As the membership function of residual energy and link quality are the same as the domain, the membership function curve of input variables is drawn depending on the above membership function equation, as shown in Figure 4.

Output variables also need to be fuzzified, using a method similar to the input variables; the specific process is as follows.

The domain of the output variable is $\mathrm{PB}=[0,1]$, quantified as $\{\mathrm{VL}, \mathrm{L}, \mathrm{M}, \mathrm{H}, \mathrm{VH}\}$ (very low, low, medium, high, very high), and triangle membership function is used. The fuzzy language of the output variable is arranged in 5 levels, which not only can cover the entire domain uniformly but also leave the output smooth. Therefore, the membership function is computed in equations (11)-(15); the membership function curve of output variables is shown in Figure 5.

$$
\begin{aligned}
& \mu_{\mathrm{VL}}(\mathrm{PB})=\left\{\begin{array}{l}
-4 \mathrm{~PB}+1,0 \leq \mathrm{PB} \leq 0.25, \\
0, \mathrm{~PB}>0.25 \& \mathrm{~PB}<0,
\end{array}\right. \\
& \mu_{\mathrm{L}}(\mathrm{PB})=\left\{\begin{array}{l}
4 \mathrm{~PB}, 0 \leq \mathrm{PB} \leq 0.25, \\
-4 \mathrm{~PB}+2,0.25 \leq \mathrm{PB} \leq 0.5, \\
0, \mathrm{~PB}<0 \& \mathrm{~PB}>0.5,
\end{array}\right.
\end{aligned}
$$




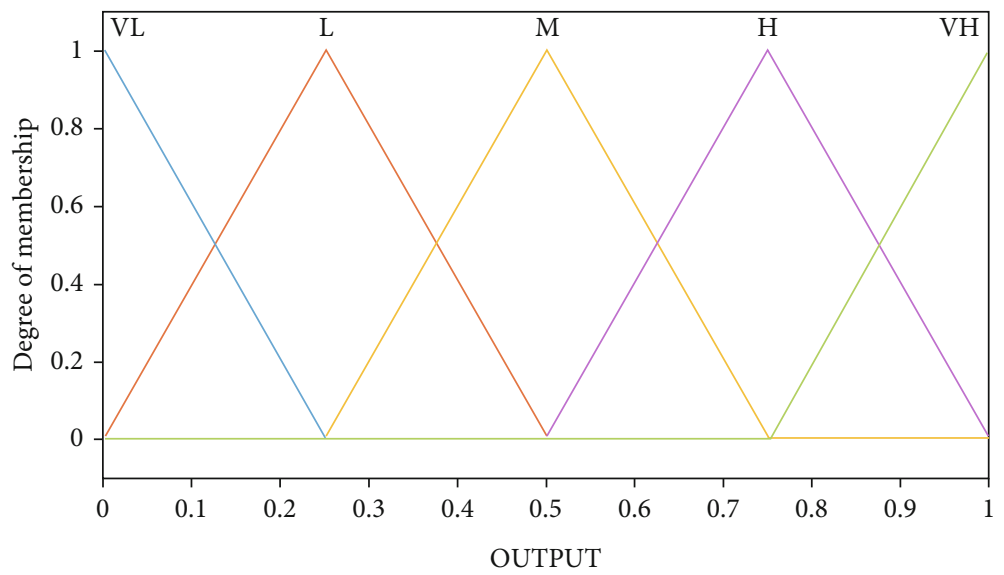

FIgURE 5: Membership function curve of output variables.

$$
\begin{aligned}
& \mu_{\mathrm{M}}(\mathrm{PB})=\left\{\begin{array}{l}
4 \mathrm{~PB}-1,0.25 \leq \mathrm{PB} \leq 0.5, \\
-4 \mathrm{~PB}+3,0.5 \leq \mathrm{PB} \leq 0.75, \\
0, \mathrm{~PB}<0.25 \& \mathrm{~PB}>0.75,
\end{array}\right. \\
& \mu_{\mathrm{H}}(\mathrm{PB})=\left\{\begin{array}{l}
4 \mathrm{~PB}-2,0.5 \leq \mathrm{PB} \leq 0.75, \\
-4 \mathrm{~PB}+4,0.75 \leq \mathrm{PB} \leq 1, \\
0, \mathrm{~PB}<0.5 \& \mathrm{~PB}>1,
\end{array}\right. \\
& \mu_{\mathrm{VH}}(\mathrm{PB})=\left\{\begin{array}{l}
3,0.75 \leq \mathrm{PB} \leq 1, \\
0, \mathrm{~PB}<0.75 \& \mathrm{~PB}>1,
\end{array}\right.
\end{aligned}
$$

where $\mu_{\mathrm{VL}}(\mathrm{PB})$ represents the membership function of $\mathrm{PB}$ belonging to $\mathrm{VL}, \mu_{\mathrm{L}}(\mathrm{PB})$ is the membership function of $\mathrm{PB}$ belonging to $\mathrm{L}, \mu_{\mathrm{M}}(\mathrm{PB})$ indicates the membership function of $\mathrm{PB}$ belonging to $\mathrm{M}, \mu_{\mathrm{H}}(\mathrm{PB})$ is the membership function of $\mathrm{PB}$ belonging to $\mathrm{H}$, and $\mu_{\mathrm{VH}}(\mathrm{PB})$ is the membership function of $\mathrm{PB}$ belonging to $\mathrm{VH}$.

4.2.2. Fuzzy Inference. After the input and output variables are fuzzified, these variables enter the fuzzy inference process. The method adopts Mamdani fuzzy inference method and formulates a set of fuzzy rules in advance depending on the relationship between fuzzy input and funny output to realize the inference calculation from input to output. The formulation of fuzzy rules usually consists of a set of fuzzy conditional sentences with If-Then structure, such as

$$
\begin{aligned}
& \text { If }(\mathrm{RE}=\mathrm{H}) \&(\mathrm{LQ}=\mathrm{L}) \text { ThenOutput }=\mathrm{H}, \\
& \text { If }(\mathrm{RE}=\mathrm{M}) \&(\mathrm{LQ}=\mathrm{L}) \text { Then Output }=\mathrm{M} .
\end{aligned}
$$

The value of path benefit value is affected by fuzzy rules. According to the different emphases on the residual energy and link quality required by the periodic data and emergency data, the corresponding fuzzy rules are separately formulated for inference; for example, the periodic packets pay more attention to the residual energy, while the emergency packets pay more attention to link quality. Table 2 shows the fuzzy
TABle 2: Fuzzy rules for periodic data.

\begin{tabular}{lccc}
\hline Rule & LQ & RE & Output \\
\hline 1 & L & L & VL \\
2 & L & M & M \\
3 & L & H & H \\
4 & M & L & L \\
5 & M & M & M \\
6 & M & H & H \\
7 & H & L & L \\
8 & H & M & H \\
9 & H & H & VH \\
\hline
\end{tabular}

TABLe 3: Fuzzy rules for emergency data.

\begin{tabular}{lccc}
\hline Rule & LQ & RE & Output \\
\hline 1 & L & L & VL \\
2 & L & M & L \\
3 & L & H & L \\
4 & M & L & M \\
5 & M & M & M \\
6 & M & H & H \\
7 & H & L & H \\
8 & H & M & H \\
9 & H & H & VH \\
\hline
\end{tabular}

rules for periodic data, and Table 3 depicts the fuzzy rules for emergency data.

4.2.3. Defuzzification. At the end of the fuzzy inference process, the fuzzy output will be obtained depending on the rules of periodic data or emergency data, no matter what kind of fuzzy output needs to be defuzzified to get the path benefit. The center of the gravity method is used as the defuzzification method. The center of gravity method takes the area center of gravity enclosed by the membership function curve 


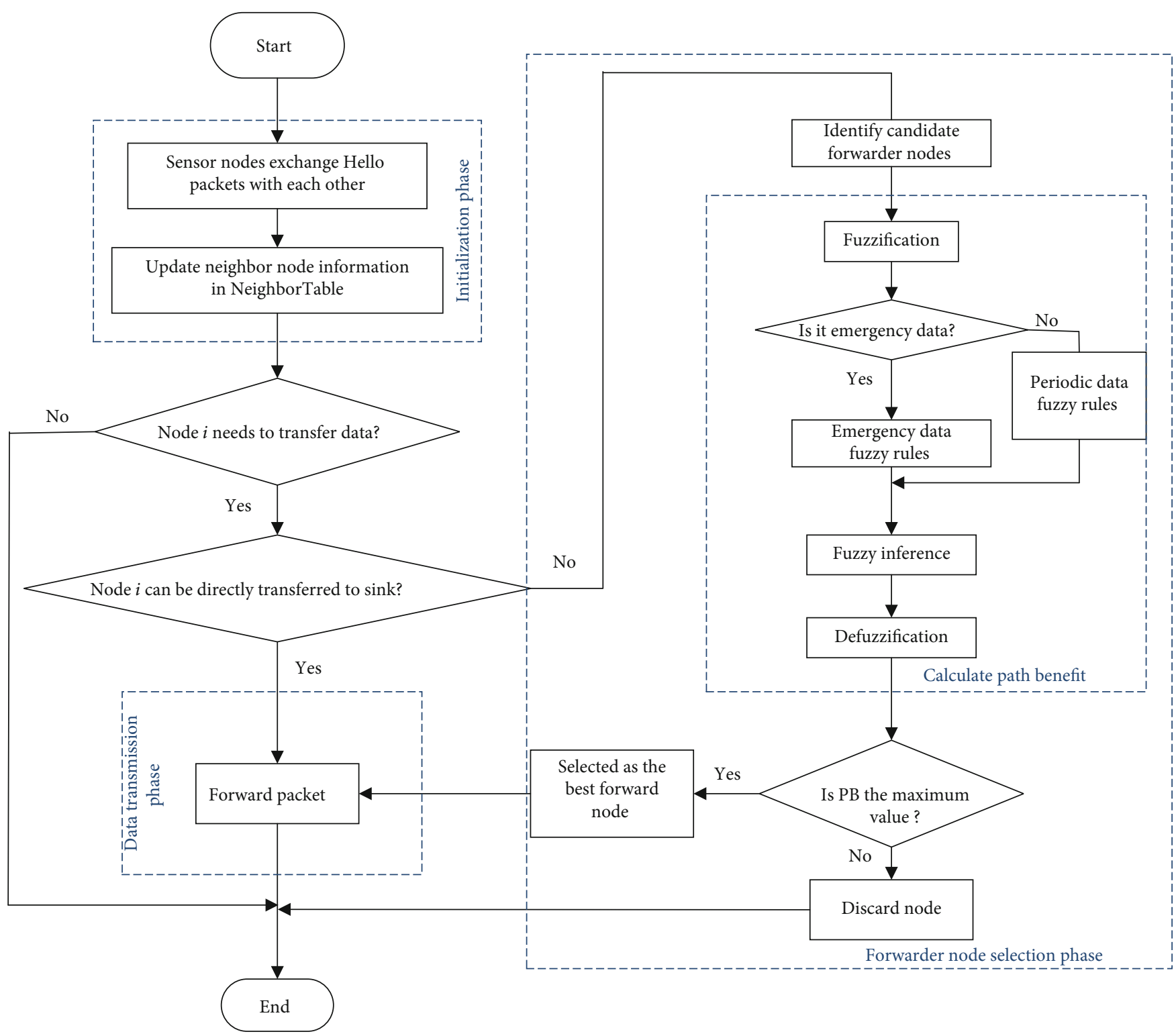

Figure 6: Flow diagram of the proposed methodology.

to calculate, which can output the reasoning value more smoothly.

Hence, the center of the gravity method is used to defuzzify the fuzzy output from fuzzy inference, and finally, the path benefit value of each candidate forwarder is obtained.

4.3. EARP: Operational Steps. The proposed protocol has three phases: initialization phase, forwarder node selection phase, and data transmission phase. Figure 6 presents the flow diagram of the proposed methodology.

4.3.1. Initialization Phase. Each sensor node periodically broadcasts Hello packets (HP) to its surrounding nodes. After any node receives HP from other neighbor nodes, it will establish or update the neighbor table (NT) information. The Hello packet includes the node ID, data type (DT), residual energy (RE), link quality (LQ), and hop count $(H)$.
Each node must timely update the current status information and its neighbor information to ensure the stable link connection and avoid the misleading of outdated information. Sensor node maintains a neighbor table, which relies on collecting Hello packet exchanged between nodes to establish or update information. The procedure for establishing and updating the neighbor table is demonstrated in Algorithm 1.

4.3.2. Forwarder Node Selection Phase. If the transmission range of the node $i$ does not support the direct transmission to sink, it is necessary to select the best forwarder node among its candidate nodes to transmit data. The candidate nodes are composed of neighbor nodes with minimum hops.

The principle of the best forwarder node selection is to establish a fuzzy control model consisting of residual energy and link quality in the candidate nodes, calculate 


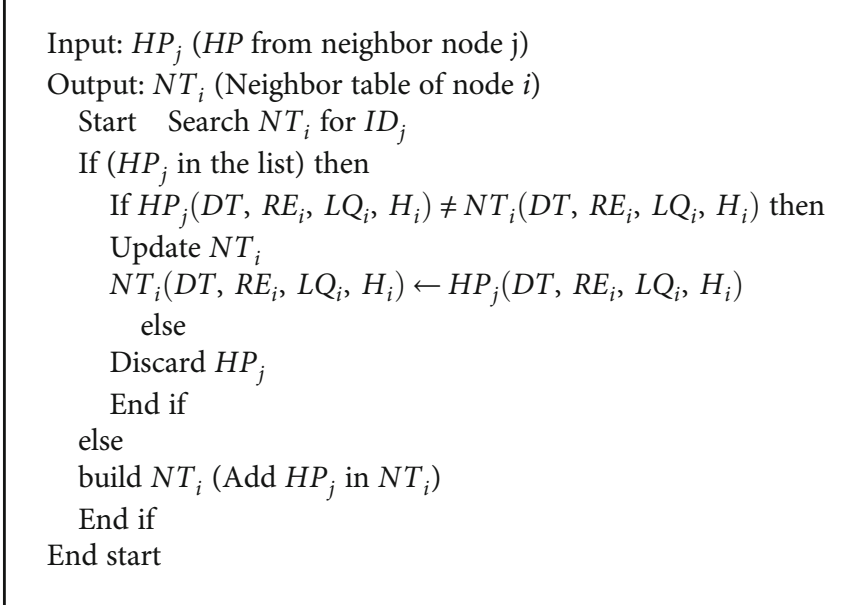

Algorithm 1: Neighbor table construction algorithm.

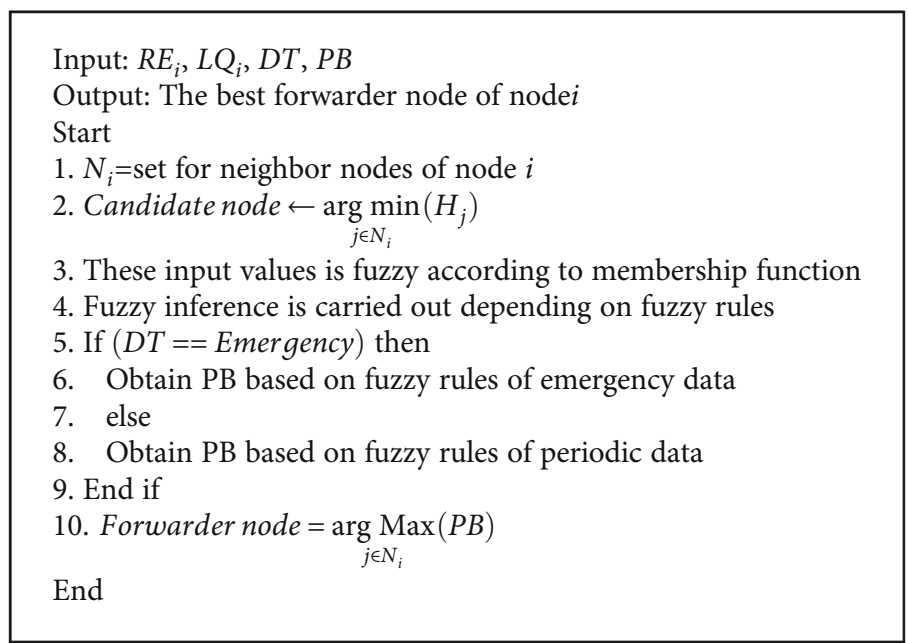

Algorithm 2: The best forwarder node selection process.

the corresponding $\mathrm{PB}$ when transmitting emergency data or periodic data, and compare the calculated $\mathrm{PB}$. The candidate forwarder node with the maximum $\mathrm{PB}$ is selected as the best forwarder node for data transmission. The best forwarder node selection process is described in Algorithm 2.

4.3.3. Transmission Phase. Once the best forwarder node is selected, data transmission begins. The source node sends the packets to the selected best forwarder node and then repeats the above steps until packets are transmitted to sink.

If the node carrying periodic data and another node carrying emergency data both select the same forwarder node, emergency data are preferentially forwarded based on the priority principle. If the remaining node energy is lower than the threshold level, only its own data is transmitted.

\section{Performance Evaluation}

5.1. Simulation Environment and Parameters. The experiment considers deploying 10 biosensors and 1 sink on the
TABLE 4: Simulation parameters.

\begin{tabular}{lc}
\hline Parameters & Values \\
\hline Initial energy & $0.5 \mathrm{~J}$ \\
Number of sensor nodes & 10 \\
Number of sink & 1 \\
Transmitting energy & $16.7 \mathrm{~nJ} / \mathrm{bit}$ \\
Receiving energy & $36.1 \mathrm{~nJ} / \mathrm{bit}$ \\
Data aggregation energy & $5 \mathrm{~nJ} / \mathrm{bit}$ \\
Amplifier energy & $1.97 e-9 \mathrm{~J} / \mathrm{b}$ \\
\hline
\end{tabular}

human body. Sink is placed at the center of the network. The transmission range of nodes is set to $30 \mathrm{~cm}$. We evaluate the performance using MATLAB 2017b simulation and compared EARP with existing routing protocols EERDT and M-TSIMPLE. Both the existing two protocols and proposed EARP protocol are based on the data transmission of forwarder node. We referred to the simulation parameters of Nordic nRF2401 [31], which is a low-power single-chip 


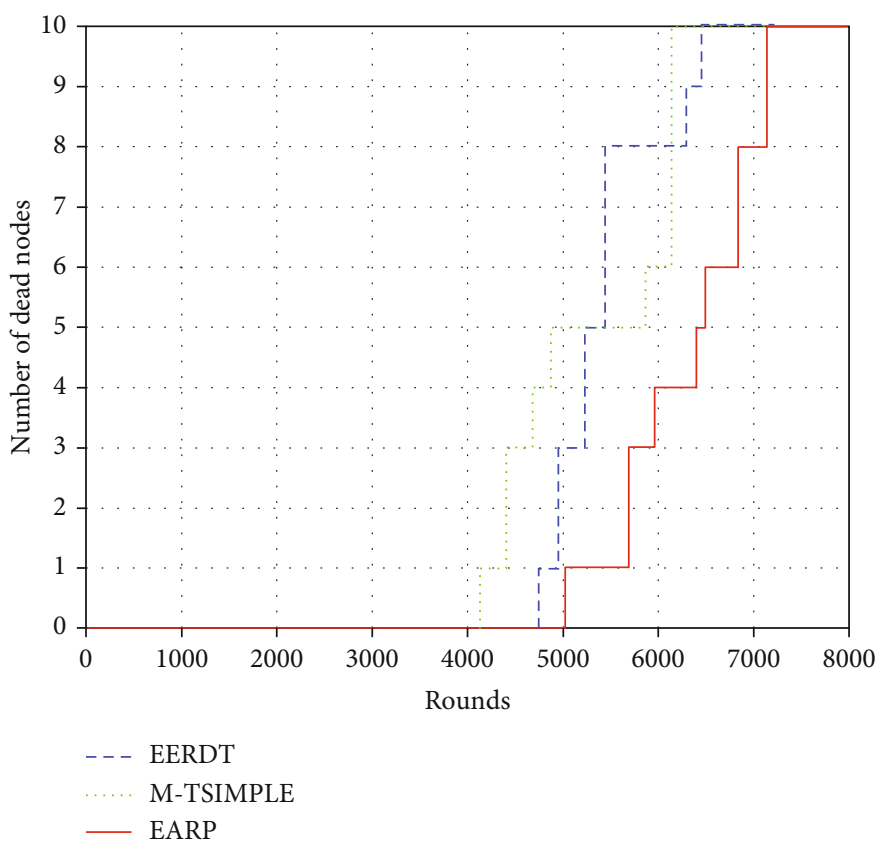

Figure 7: Network stability period and lifetime comparison.

transceiver commonly used in body sensor networks. The simulation parameter values are summarized in Table 4. The performance of the EARP protocol is measured based on network lifetime, remaining energy, throughput, and packet delivery ratio.

5.2. Simulation Analysis. The network lifetime is expressed as the time from the network start to the death of the last node. The network stability period refers to the time from the network start to the death of the first node. Figure 7 displays the comparison of EARP, EERDT, and M-TSIMPLE in network lifetime. Compared with EERDT and M-TSIMPLE, EARP found that the first death node appeared in 5030 rounds, while EERDT and M-TSIMPLE die at 4750 rounds and 4130 rounds, respectively. The entire nodes of EERDT and M-TSIMPLE die at 6450 and 6130 rounds, respectively, while the EARP protocol is able to live up to 7150 rounds.

With regard to a complex WBAN, the more parameters considered, the more difficult it is to accurately describe the dynamic characteristics of the network, while the fuzzy control model added in EARP can better adapt to the characteristics of WBAN. Moreover, EARP adapts the fuzzy control model to calculate the path benefit of multiple parameters. Compared with the other two protocols, the computational complexity of EARP is higher, which leads to increase the calculation time and corresponding energy consumption. However, EARP protocol has better performance in network lifetime than the existing two protocols, mainly because EARP considers the link quality when selecting the best forwarder; the reliability of the selected path is higher than that of EERDT and M-TSIMPLE, which avoids the energy consumed by retransmission after packet loss. EERDT balances the network energy consumption through different transmission methods, so its network lifetime is higher than
M-TSIMPLE. However, M-TSIMPLE regards the mobility of the human body, because its network topology is unstable and the link quality is not considered; it would cause data transmission failure, so the data retransmission consumes a lot of energy.

Using the residual energy to analyze the energy efficiency in the network, Figure 8 describes the energy consumption comparison between EARP, EERDT, and M-TSIMPLE. As the number of rounds increases, the average residual energy gradually decreases. As shown in Figure 8, the energy consumption of EARP is more than half at 3100 rounds, while EERDT and M-TSIMPLE are more than half at 2300 and 1700 rounds, respectively.

The analysis reveals that EARP has less energy consumption than EERDT and M-TSIMPLE, because EARP uses the fuzzy control model to quickly establish routing path and plans corresponding transmission paths in conformity with data types to balance network energy consumption. EERDT adopts a cluster-based approach to select forwarder node, and the initial energy of the cluster-head is the same as that of other nodes, which would cause the cluster-head to consume its energy rapidly because it has been selected as the forwarder node for many times. EERDT transmits emergency data and normal data in the way of single hop and multihop coexisting, while the unstable network topology of M-TSIMPLE is easy to retransmit packets, which results in excessive energy consumption of nodes, so its energy efficiency is lower than EERDT.

Throughput represents the total number of packets successfully received by sink, and its change depends on the number of sensors alive in the network. Figure 9 presents the throughput analysis of EARP, EERDT, and $M-$ TSIMPLE. With the increase of rounds, the longer the network stability period is, the more packets will be delivered 


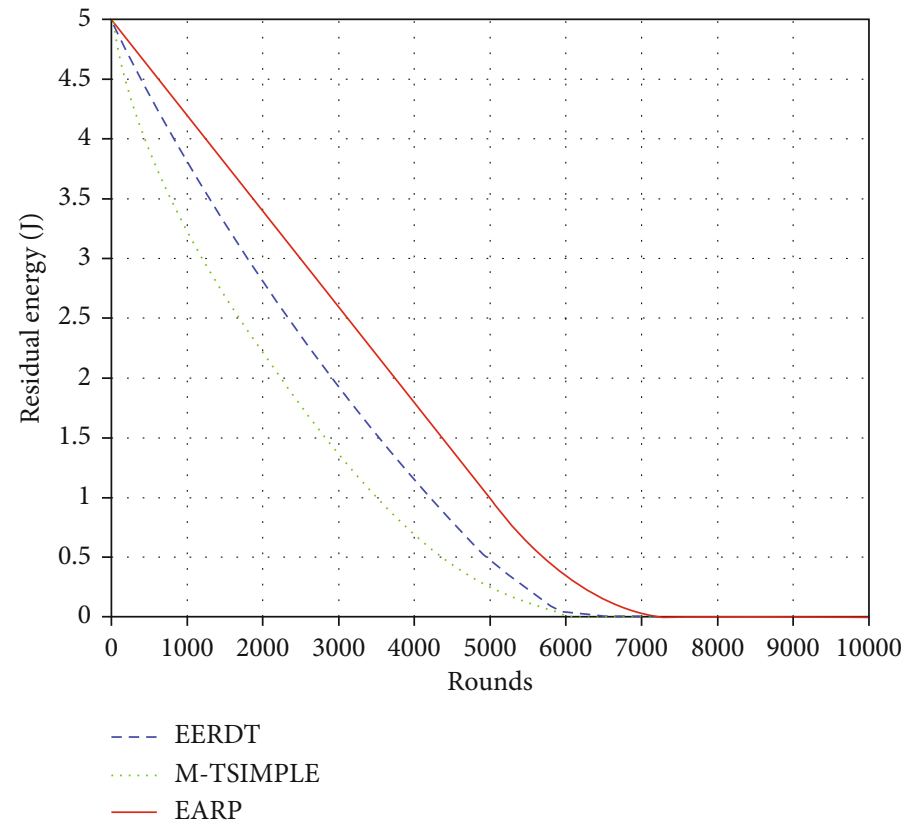

FIGURE 8: Energy consumption.

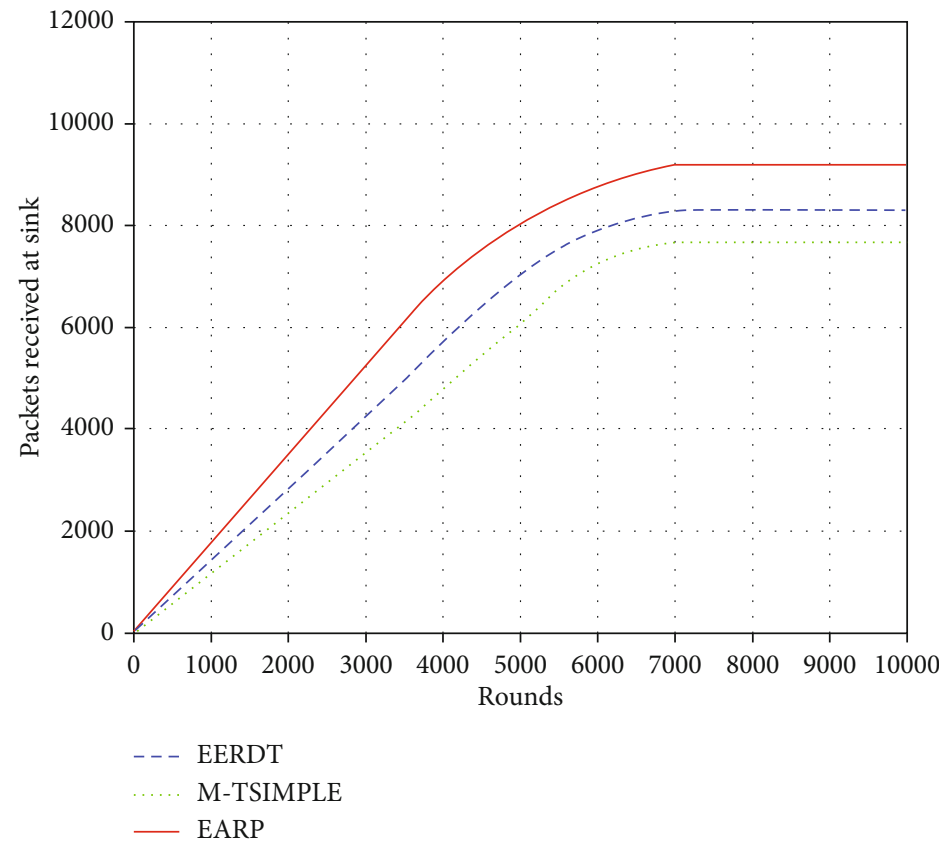

FIGURE 9: Network throughput.

in the whole network. Compared with the existing two protocols, EARP has obvious advantages in throughput. EARP exploits a fuzzy control model to quickly select forwarder node and establish routing paths, which can increase the throughput of the entire WBAN. Moreover, EARP has the longest network lifetime compared to EERDT and MTSIMPLE. The longer the network lifetime, the more packets can be transmitted and received. The throughput of EERDT is relatively higher than that of M-TSIMPLE, because EERDT uses the combination of single hop and multihop to transmit packets for the improvement of the stability of the link, so its network stability period is longer than that of M-TSIMPLE.

The packet delivery ratio is a central parameter to measure the reliability of the routing protocol. The packet delivery ratio represents the ratio of the number of data packets sent by the source node to the number of data packets received by sink. As the number of rounds increases, the number of dead nodes also increases, which affects the packet delivery ratio. Figure 10 depicts the analysis of EARP, EERDT, and M-TSIMPLE in the packet delivery ratio. 


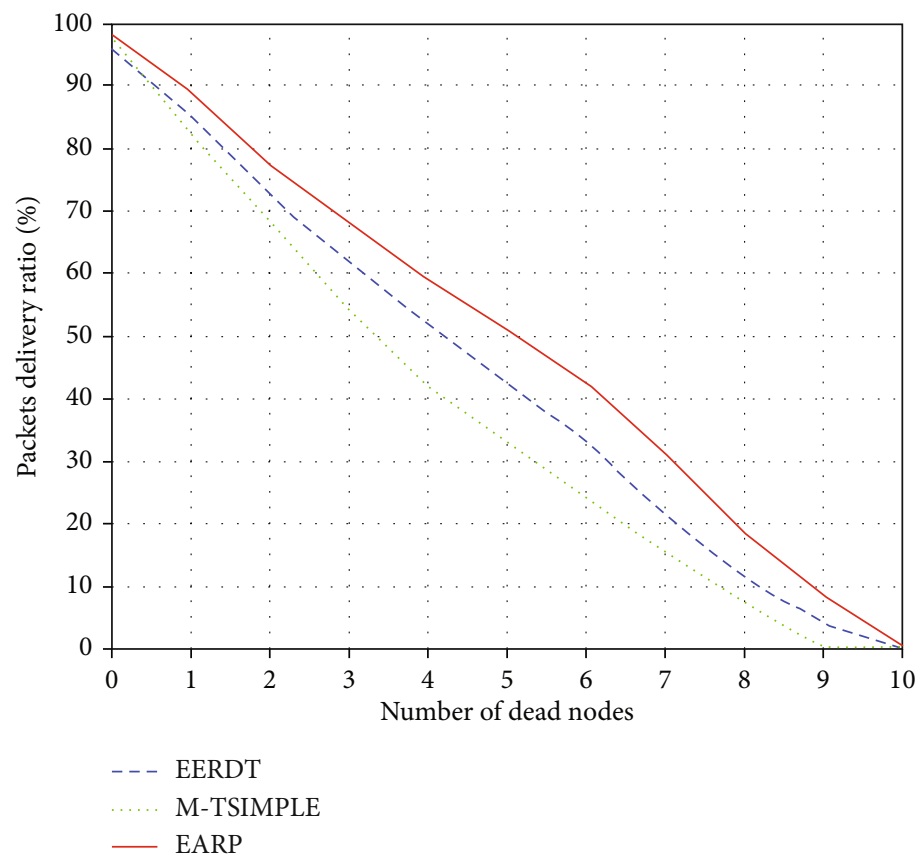

Figure 10: Packet delivery ratio.

Compared with EERDT and M-TSIMPLE, EARP has a higher packet delivery ratio, because it considers link quality parameter when establishing an effective routing path, while EERDT and M-TSIMPLE do not consider the impact of link quality on reliability. Furthermore, EARP transmits packets depending on priority, when available nodes are reduced; packet orderly transmission can avoid packet loss. EERDT performs better than M-TSIMPLE, which is due to the fact that EERDT does not analyze the dynamic network characteristics, so the data transmission is more stable than M-TSIMPLE.

\section{Conclusion}

In the current work, this paper proposes a fuzzy control based energy-aware routing protocol, which is used to effectively select forwarder node in WBANs. Longer network lifetime and timely reliability of emergency data delivery are the basic requirements for the smooth operation of WBAN. The proposed protocol divides the data into emergency data and periodic data depending on the importance of the data and uses the fuzzy control model which consists of residual energy and link quality to select the appropriate path for the two data transmissions, respectively. This method satisfies the different QoS requirements for WBAN. Compared with the simulation results of the existing EERDT and $\mathrm{M}$ TSIMPLE, the proposed EARP performs well in network lifetime, remaining energy, throughput, and packet delivery rate.

Data transmission in WBAN leads to continuous energy consumption. Energy harvesting technology (kinetic energy or thermal energy generated by the human body is converted into electrical energy) is used to supplement energy for energy-poor WBAN. In the future, we will try to introduce green energy technology to achieve better energy-saving effect.

\section{Data Availability}

No data have to be included.

\section{Conflicts of Interest}

The authors declare that there is no conflict of interest regarding the publication of this paper.

\section{Acknowledgments}

This work was supported in part by the National Natural Science Foundation of China $(61772175,61801170,62072158$, and 61501405), in part by the Key Science and Research Program in the University of Henan Province (under Grant 21A510001), in part by the Science \& Technology Major Program of Henan Province of China (161100210900), in part by the International Cooperation Foundation of Henan Province (172102410072), in part by the Science and Technology Research Project of Henan Province (under Grant 182102210285), in part by the Postdoctoral Science Foundation of China (under Grants 2018M632772 and 2018M633351), and in part by the program of Science and Technology of Luoyang City (1901016A).

\section{References}

[1] G. Fortino, R. Giannantonio, R. Gravina, P. Kuryloski, and R. Jafari, "Enabling effective programming and flexible management of efficient body sensor network applications," IEEE Transactions on Human-Machine Systems, vol. 43, no. 1, pp. 115-133, 2013.

[2] M. Chen, S. Gonzalez, A. V. Vasilakos, H. Cao, and V. C. M. Leung, "Body area networks: a survey," Mobile Networks and Applications, vol. 16, no. 2, pp. 171-193, 2011. 
[3] R. Punj and R. Kumar, "Technological aspects of WBANs for health monitoring: a comprehensive review," Wireless Networks, vol. 25, no. 3, pp. 1125-1157, 2019.

[4] R. Kumari and P. Nand, "Performance comparison of various routing protocols in WSN and WBAN," in 2016 International Conference on Computing, Communication and Automation (ICCCA), pp. 427-431, Noida India, April 2016.

[5] R. Negra, I. Jemili, and A. Belghith, "Wireless body area networks: applications and technologies," Procedia Computer Science, vol. 83, pp. 1274-1281, 2016.

[6] C. Savaglio, P. Pace, G. Aloi, G. Fortino, and G. Fortino, "Lightweight reinforcement learning for energy efficient communications in wireless sensor networks," IEEE Access, vol. 7, pp. 29355-29364, 2019.

[7] G. Smart, N. Deligiannis, and R. Surace, "Decentralized timesynchronized channel swapping for ad hoc wireless networks," IEEE Transactions on Vehicular Technology, vol. 65, no. 10, pp. 8538-8553, 2016.

[8] C. Oey and S. Moh, "A survey on temperature-aware routing protocols in wireless body sensor networks," Sensors, vol. 13, no. 8, pp. 9860-9877, 2013.

[9] T. I. ul Huque, K. S. Munasinghe, M. Abolhasan, and A. Jamalipour, "EAR-BAN: energy efficient adaptive routing in wireless body area networks," in Proceedings of the 7th International Conference on Signal Processing and Communication Systems (ICSPCS), pp. 121-139, Carrara, VIC, Australia, December 2013.

[10] A. Kurian and R. Divya, "A survey on energy efficient routing protocols in wireless body area networks (WBAN)," in International Conference on Innovations in Information, Embedded and Communication Systems (ICIIECS), pp. 1-6, Coimbatore, India, March, 2017.

[11] M. Asif, S. Khan, and R. Ahmad, "Quality of service of routing protocols in wireless sensor networks: a review," IEEE Access, vol. 5, pp. 1846-1871, 2017.

[12] G. Ahmed, Z. Jianhua, and M. M. S. Fareed, "PERA: prioritybased energy-efficient routing algorithm for WBANs," Wireless Personal Communications, vol. 96, no. 3, pp. 4737-4753, 2017.

[13] K. N. Qureshi, A. H. Abdullah, F. Bashir, S. Iqbal, and K. M. Awan, "Cluster-based data dissemination, cluster head formation under sparse, and dense traffic conditions for vehicular ad hoc networks," International Journal of Communication Systems, vol. 31, no. 8, p. e3533, 2018.

[14] N. Yessad, M. Omar, A. Tari, and A. Bouabdallah, "QoS-based routing in wireless body area networks: a survey and taxonomy," Computing, vol. 100, no. 3, pp. 245-275, 2018.

[15] Y. Qu, G. Zheng, H. Ma, X. Wang, B. Ji, and H. Wu, "A survey of routing protocols in WBAN for healthcare applications," Sensors, vol. 19, no. 7, p. 1638, 2019.

[16] A. Ahmad, N. Javaid, U. Qasim, M. Ishfaq, Z. A. Khan, and T. A. Alghamdi, "RE-ATTEMPT: a new energy-efficient routing protocol for wireless body area sensor networks," International Journal of Distributed Sensor Networks, vol. 10, no. 4, Article ID 464010, 2014.

[17] S. Singh, S. Negi, and A. Uniyal, "Modified new-attempt routing protocol for wireless body area network," in 2nd International Conference on Advances in Computing, Communication, \& Automation (ICACCA), pp. 1-5, Bareilly, India, October 2016.

[18] E. Selem, M. Fatehy, S. M. Abd el-Kader, and H. Nassar, “THE (temperature heterogeneity energy) aware routing protocol for
IoT health application," IEEE Access, vol. 7, pp. 108957108968, 2019.

[19] Z. A. Khan, S. Sivakumar, and W. Phillips, "ZEQoS: a new energy and QoS-aware routing protocol for communication of sensor devices in healthcare system," International Journal of Distributed Sensor Networks, vol. 10, Article ID 627689, 2014.

[20] N. Javaid, Z. Abbas, M. Fareed, Z. A. Khan, and N. Alrajeh, "M-ATTEMPT: a new energy-efficient routing protocol for wireless body area sensor networks," Procedia Computer Science, vol. 19, pp. 224-231, 2013.

[21] M. Effatparvar, M. Dehghan, and A. M. Rahmani, "A comprehensive survey of energy-aware routing protocols in wireless body area sensor networks," Journal of Medical Systems, vol. 40, no. 9, p. 201, 2016.

[22] N. Javaid, A. Ahmad, Q. Nadeem, M. Imran, and N. Haider, "IM-SIMPLE: iMproved stable increased-throughput multihop link efficient routing protocol for wireless body area networks," Computers in Human Behavior, vol. 51, pp. 1003 1011, 2015.

[23] S. M. Moid, J. Nadeem, and I. Muhammad, "BEC: a novel routing protocol for balanced energy consumption in wireless body area networks," in 11th IEEE International Wireless Communications and Mobile Computing Conference (IEEE IWCMC), pp. 653-658, Dubrovnik, Croatia, August 2015.

[24] D. Sethi and P. P. Bhattacharya, "A study on energy efficient and reliable data transfer (EERDT) protocol for WBAN," in 2nd International Conference on Computational Intelligence \& Communication Technology (CICT), pp. 254-258, Ghaziabad, India, February 2016.

[25] V. Navya and P. Deepalakshmi, "Mobility supported threshold based stability increased throughput to sink using multihop routing protocol for link efficiency in wireless body area networks (M-TSIMPLE)," in 2017 IEEE International Conference on Intelligent Techniques in Control, Optimization and Signal Processing (INCOS), pp. 1-7, Srivilliputhur, India, March 2017.

[26] M. Anwar, A. Abdullah, A. Altameem et al., "Green communication for wireless body area networks: energy aware link efficient routing approach,” Sensors, vol. 18, no. 10, p. 3237, 2018.

[27] K. N. Qureshi, F. Bashir, and A. H. Abdullah, "An energy and link aware next node selection protocol for body area networks," in 2018 International Conference on Information Networking (ICOIN), pp. 721-726, Chiang Mai, Thailand, January 2018.

[28] R. A. Isabel and E. Baburaj, "An optimal trust aware cluster based routing protocol using fuzzy based trust inference model and improved evolutionary particle swarm optimization in WBANs," Wireless Personal Communications, vol. 101, no. 1, pp. 201-222, 2018.

[29] K. N. Qureshi, S. Din, G. Jeon, and F. Piccialli, "Link quality and energy utilization based preferable next hop selection routing for wireless body area networks," Computer Communications, vol. 149, pp. 382-392, 2020.

[30] B. Manzoor, N. Javaid, A. Bibi, Z. A. Khan, and M. Tahir, "Channel modeling and energy utilization in wireless body area networks," in Proceedings of the 2012 IEEE 14th International Conference on High Performance Computing and Communication \& 2012 IEEE 9th International Conference on Embedded Software and Systems, Liverpool, UK, June, 2012.

[31] N. Semiconductor, Single Chip 2.4 GHz Transceiver Product Specification, Nordic Semiconductor ASA, Trondheim, Norway, 2007. 